4. Lovecchio F, Berlin R, Brubacher JR, Sholar JB. Hypertonic sodium bicarbonate in an acute flecainide overdose. Am J Emerg Med 1998; 16: 534-7.

5. Ranger S, Sheldon R, Fermini B, et al. Modulation of flecainide's cardiac sodium channel blocking actions be extracellular sodium: A possible cellular mechanism for the action of sodium salts in flecainide cardiotoxicity. J Pharmacol Exp Ther 1993; 264: 1160-7.

6. Sagie A, Strasberg B, Kusniec J, Sclarovsky S, Rechavia E, Agmon J. Rapid suppression of flecainide-induced incessant ventricular tachycardia with high dose intravenous amiodarone. Chest 1988; 93: 879-80.

7. Siegers A, Board PN. Amiodarone used in successful resucitation after near-fatal flecainide overdose. Resucitation 2002; 53: 105-8.

8. Maury P, Vuille C, Metzger J, Veragut B, Schoenenberger I, Elamly A, Chevrolet JC. Intoxication severe a l'acetate de flecainide. A propos d'un cas. Arch Mal Coeur Vaiss 1999; 92: 273-7.

\section{Pseudoaneurisma postinfarto del ventrículo izquierdo: hallazgos en resonancia magnética}

\section{Sr. Director:}

El pseudoaneurisma es una complicación poco frecuente en relación con el infarto agudo de miocardio. Suele ser un hallazgo casual en las técnicas de imagen. La resonancia magnética es utilizada con mayor frecuencia, dado que permite obtener datos morfológicos y funcionales de las cavidades cardiacas.

Presentamos el caso de una paciente mujer de 66 años de edad con cardiopatía isquémica tipo infarto agudo de miocardio inferolateral en diciembre de 2000 , con afectación del ventrículo derecho, que se complicó con una comunicación interventricular (CIV). En cateterismo cardiaco se objetivó un origen anómalo del tronco coronario izquierdo en la arteria coronaria derecha, con lesión del tronco coronario izquierdo del $70 \%$, que precisó cirugía de revascularización coronaria con arteria mamaria interna a primera diagonal y cierre de la CIV. En estudio por resonancia magnética cardiaca (Fig. 1) se observó un adelgazamiento de la pared inferior del ventrículo izquierdo asociado a un defecto en su pared en comunicación con una lesión contenida por el pericardio con flujo en su interior a través del defecto de la pared y en interior de la lesión, en relación con un pseudoaneurisma. La paciente ha sido intervenida con implante tipo oclusión, permaneciendo estable clínicamente y por ecocardiografía.

La rotura de la pared libre del ventrículo izquierdo es una complicación catastrófica del infarto de miocardio, que ocurre aproximadamente en un $4 \%$ de pacientes y en un $23 \%$ de los que sufren infarto fatal $(1,2)$. En la mayor parte de los casos, dicha rotura cardiaca se asocia con muerte súbita por hemopericardio y taponamiento cardiaco $(1,3,4)$. Cuando la rotura de la pared libre del ventrículo izquierdo está contenida por el pericardio adyacente se denomina pseudoaneurisma. Habitualmente es un descubrimiento incidental en ecocardiografía u otras técnicas de imagen. También pueden condicionar un fallo cardiaco congestivo por la no contractilidad de la cavidad o provocar embolismos por retención de flujo sanguíneo en su interior $(1,4)$.

Tanto aneurismas como pseudoaneurismas del corazón son secuelas del infarto de miocardio. Un aneurisma ventricular verdadero es una complicación crónica del infarto de miocardio y son áreas de miocardio cicatricial, adelgazado, con motilidad disquinética. Un saco aneurismático verdadero contiene endocardio, epicardio y tejido fibroso fino (cicatriz) que es un remanente del músculo ventricular, mientras que el saco del pseudoaneurisma representa pericardio que contiene a un ventrículo izquierdo roto. Un aneurisma pequeño puede no causar síntomas y es compatible con una supervivencia prolongada, con un bajo potencial de rotura comparado con el pseudoaneurisma. Pero también pueden desarrollar fallo cardiaco con-

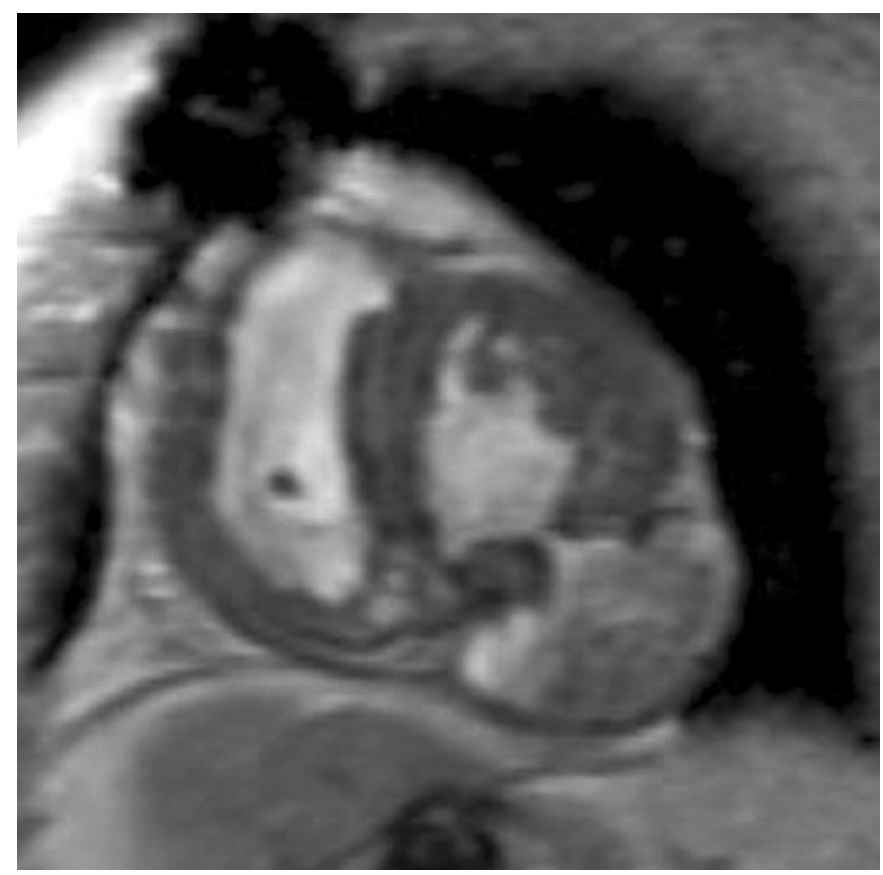

Fig. 1. Resonancia magnética. Eje corto ventrículos gradiente T2.

gestivo, embolismos y arritmias ventriculares debido a los efectos de la cicatriz ventricular en la conducción cardiaca. Mientras los pseudoaneurismas requieren un tratamiento quirúrgico urgente por su posibilidad de ruptura, los aneurismas verdaderos pueden ser tratados médicamente y únicamente es necesaria su reparación quirúrgica si se asocia con angina refractaria, fallo cardiaco congestivo, embolismo sistémico o arritmias refractarias (1-3).

La presencia de miocardio rodeando la cavidad sugiere un aneurisma verdadero y la discontinuidad del miocardo pseudoaneurisma. La ecografia Doppler color será de gran utilidad si no es posible demostrar la discontinuidad del miocardio. Así, la relación orificio de entrada-diámetro máximo de la cavidad (mayor en aneurismas), las características del flujo en ecografía Doppler color y pulsado en la cámara (presencia de flujo turbulento en el cuello o en el interior de la cavidad y flujo bidireccional en ecografía Doppler color y pulsado sugieren pseudoaneurisma) (5) y la relación de las arterias coronarias con la cámara deben ser usadas para la identificación de la lesión (pseudoaneurisma: la cavidad se situa fuera del epicardio, aneurisma: no se extiende fuera de las arterias coronarias al estar rodeado por epicardio) (6).

En las técnicas de diagnóstico por imagen, además de la ecografía Doppler color, la resonancia magnética tiene un potencial significativo en la evaluación no invasiva de pacientes con infarto de miocardio y sus complicaciones (7). Puede localizar el aneurisma, distinguir entre pericardio, trombo y miocardio. Un estudio de viabilidad miocárdica por resonancia magnética utiliza la administración intravenosa de contraste paramagnético de forma diferida, lo que permite delimitar el tamaño del infarto y su extensión. En el caso de un aneurisma verdadero el tejido que constituye la pared del aneurisma presentará realce diferido, indicativo de tejido cicatricial a consecuencia del músculo miocárdico infartado, mientras el saco de un pseudoaneurisma no se realzará por ser pericardio $(7,8)$.

\section{A. Vara Castrodeza, A. Casero Lambas ${ }^{1}$, T. Escudero Caro, M. Mendo González}

Servicios de Radiodiagnóstico y ${ }^{1}$ Cardiología. Hospital Universitario Río Hortega. Valladolid 
1. Brown SL, Gropler RJ, Harris KM. Distinguishing left ventricular aneurysm from pseudoaneurysm. A review of the literature. Chest 1997; 111: 1403-9.

2. Pollak H, Nobis H, Miczoch J. Frequency of left ventricular free wall rupture complicating acute myocardial infarction since the advent of thrombolysis. Am J Cardiol 1994; 74: 184-6.

3. Bates RJ, Beutler S, Resnekov L, et al. Cardiac rupture-challenge in diagnosis and management. Am J Cardiol 1977; 40: 429-47.

4. Hung MJ, Wang CH, Cherng WJ. Unruptured left ventricular pseudoaneurysm following myocardial infarction. Heart 1998; 80: 94-7.

5. Gatewood RP, Nanda NC. Differentiation of left ventricular pseudoaneurysm from true aneurysm with two-dimensional echocardiography. Am J Cardiology 1980; 46: 869-78.

6. Spindola-Franco H, Kronacher N. Pseudoaneurysm of the left ventricule: radiographic and angiocardiographic diagnosis. Radiology 1978; 127: 29-34.

7. Chakraborty RN, Nicholson AA, Alamgir MF. Magnetic Resonance Images of left ventricular pseudoaneurysm. Heart 1998; 80: 101-3.

8. Kumbasar B, Wu KC, Kamel IR, Lima JAC, Bluemke DA. Left ventricular true aneurysm: diagnosis of myocardial viability shown on MR imaging. AJR 2002; 179: 472-4.

\section{Disnea aguda y TAC helicoidal torácico}

\section{Sr. Director:}

El diagnóstico de tromboembolismo pulmonar (TEP) sigue siendo complicado. Supone unos 600.000 nuevos pacientes/año en EE.UU. La angiografía pulmonar (técnica patrón) es invasiva aunque con escasas complicaciones. La gammagrafía pulmonar (GP) de alta probabilidad indica habitualmente su presencia pero pocos pacientes la presentan. Su valor predictivo positivo es del $85-87 \%$. Se altera por la presencia de EPOC o TEP previo. A continuación exponemos la utilidad de la tomografía axial computarizada helicoidal (TACH) en el diagnóstico de TEP.

Mujer de 72 años con neoplasia mamaria izquierda intervenida y tratada con quimioterapia y radioterapia 4 años antes. Dos meses antes sufrió paraparesia secundaria a compresión medular metastásica en L2. Acude por disnea brusca y sudoración. Exp. Física: TA 150/90 mm Hg, taquipnea con $34 \mathrm{rpm}$, taquicardia a $113 \mathrm{lpm}$ y roncus dispersos pulmonares. Hemograma: Hb 11,9 g/L. Bioquímica sérica normal. TP $78 \%$ con TTPa normal. Dímero D positivo [2326 ng/ml (normal 0-500)]. Gasometría arterial: $\mathrm{pO}_{2} 53 \mathrm{~mm} \mathrm{Hg}$ y $\mathrm{pCO}_{2} 27 \mathrm{mmHg}$. EKG: taquicardia sinusal con $\mathrm{S} 1$. Rx. Tórax normal. Se realizó TACH torácica que mostró la presencia de una gran trombo en la bifurcación de la arteria pulmonar extendiéndose por en nacimiento de ambas arterias pulmonares principales (Fig. 1). La GP mostró amplios defectos bilaterales de perfusión con ventilación normal. Se inició anticoagulación con lenta pero buena evolución clínica.

El diagnóstico de TEP sigue siendo difícil. La TACH lo ha revolucionado al permitir realizarlo salvo en los casos de TEP subsegmentarios $(1,2)$. Se requiere la opacificación intensa de las arterias pulmonares. El diagnóstico en las arterias centrales tiene una sensibilidad del $90 \%$ y especificidad del 96\%. Cuando se incluyen vasos subsegmentarios la sensiblidad baja al $67 \%$ y la especificidad al 89\%. La tasa de TEP sugsegmentario aislado es sólo del 6\% (3) y su importancia clínica aún no ha sido claramente definida. Parece existir un riesgo aumentado de recurrencia en los pacientes que lo padecen (4). La TACH es útil para evaluar pacientes con enfermedad cardiopulmonar grave asociada pero la seguridad de retirar anticoagulación si es negativa para TEP aún no está demostrada (5). Con la TACH de múltiples hélices para eliminar artefactos, las arterias pulmonares se pueden estudiar hasta llegar al $5^{\circ}$ orden. Esto implica una mejor probabilidad de

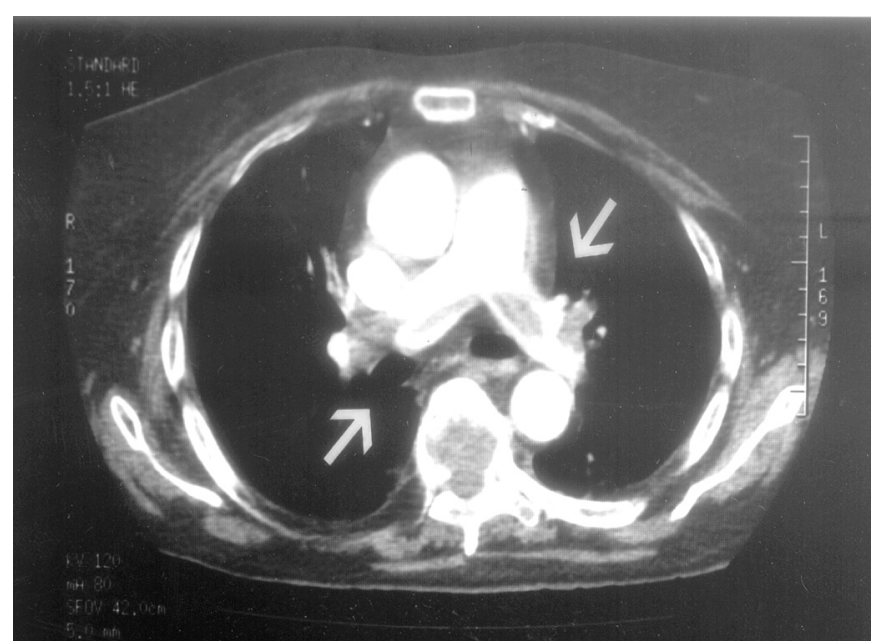

Fig. 1. TAC helicoidal torácico: presencia de un trombo en la bifurcación de arteria pulmonar.

detección de émbolos subsegmentarias (6). Así, la TACH (en especial con fase venosa) puede llegar a ser de primera línea en la evaluación del TEP (7). Una TACH negativa más una ecografía de troncos venosos también negativa incrementa sensibilidad y especificidad para TEP con respecto a la TACH sola. La GP parece más útil en pacientes previamente sanos con radiografía de tórax normal mientras que en pacientes que no cumplen esto sería más útil la TACH (8). En un estudio español se evidenció concordancia entre la GP y la TACH en el 70,3\% de los pacientes (diagnóstico de TEP 13 casos y ausencia 25). En los estudios discordantes se evidenció TEP con GP en 2 pacientes con TAC normal (9). La TACH detecta todos los TEP relevantes y ayuda al diagnóstico diferencial si la GP no es diagnóstica o de baja-intermedia probabilidad. Por otro lado, una determinación rápida de dímero D por ELISA puede reducir la necesidad de realizar una TACH en un 25\%-35\% de casos (10). La TACH es más barata, segura, rápida y fácil de hacer que la arteriografía pulmonar y además permite una mayor accesibilidad diagnóstica para el paciente urgente y en hospitales que no disponen de la posibilidad de realizar GP.

\section{J. A. Díaz Peromingo, R. Puerta Louro, E. Padín Paz, H. Periscal Martínez ${ }^{1}$}

Servicios de Medicina Interna $y^{\prime}$ Urgencias. Hospital Da Barbanza. Riveria. A Coruña

1. Gulsun M, Goodman L, Washington L. Venous thoromboembolic disease: where does multidetector computed tomography fit? Cardiol Clin 2003; 21: 631-638.

2. Weg J. Current diagnoss techniques for pulmonary embolism. Semin Vasc Surg 2000; 13: 182-188.

3. The PIOPED investigators. Value of ventilation/perfusion scan in the acute pulmonary embolism: results of the prospective investigation pulmonary embolism diagnosis (PIOPED). JAMA 1990; 263: 2753-2759.

4. Kearon C. Diagnosis of pulmonary embolism. CMAJ 2003; 168: 183194.

5. Elliott C. Pulmonary embolism diagnosis in hospitalized and intensive care unit patients. Semin Vasc Med 2001; 1: 205-221.

6. Pattynama P, Kuiper J. Second-generation, subsecond multislice computed-tomography: advancing the role of helical CT pulmonary angiography in suspected pulmonary embolism. Semin Vasc Med 2001; 1: 195-204.

7. Srivastava S, Eagleton M, Greenfield L. Diagnosis of pulmonary ambolism with various imaging modalities. Semin Vasc Surg 2004; 17 (2): 173-180. 